\title{
Comparison of in vivo cardiac function with ex vivo cardiac performance of the rat heart after thoracic irradiation
}

\author{
${ }^{1,2} \mathrm{~N}$ A P FRANKEN, ${ }^{3} \mathrm{~J}$ A J CAMPS, ${ }^{1} \mathrm{~F} \mathrm{~J} \mathrm{M}$ VAN RAVELS, ${ }^{4} \mathrm{~A}$ VAN DER LAARSE, \\ ${ }^{3} \mathrm{E} K \mathrm{~K}$ PAUWELS and ${ }^{1} \mathrm{~J}$ WONDERGEM
}

Departments of ${ }^{1}$ Clinical Oncology, ${ }^{3}$ Diagnostic Radiology and Nuclear Medicine, and ${ }^{4}$ Cardiology, University Hospital Leiden, and ${ }^{2}$ Department of Radiotherapy, Academic Medical Center, University of Amsterdam, The Netherlands

\begin{abstract}
The aim of the study was to compare in vivo cardiac function with ex vivo cardiac performance after local heart irradiation in the same rat. Left ventricular ejection fraction (LVEF) was measured in vivo by radionuclide ventriculography in Sprague-Dawley rats up to 16 months after a single dose of $20 \mathrm{~Gy}$. Four days after in vivo measurements, cardiac performance was determined ex vivo, using the isolated working rat heart preparation. After irradiation, cardiac performance measured ex vivo deteriorated more rapidly than the in vivo measured LVEF. Within 4 months post-treatment, ex vivo cardiac output and stroke volume started to decrease and declined continuously throughout the observation period of 16 months. The reduction in stroke volume was already significant $(p<0.04)$ at 4 months post-treatment, whereas the decline in cardiac output was significant $(p<0.05)$ at 12 months post-treatment. In vivo, no change in LVEF was observed during the first 12 months post-treatment. Thereafter, LVEF decreased rapidly from $65 \pm 2 \%$ to $46 \pm 8 \%(p<0.01)$, at 16 months post-treatment. Up to 12 months post-irradiation, LVEF was not correlated to ex vivo cardiac output. At 16 months post-treatment, when clinical symptoms of heart failure become evident, a positive relation between both parameters was found. The lack of correlation between the in vivo and ex vivo measurements of cardiac function during the first 12 months post-treatment might be explained by the involvement of compensatory mechanisms being operative in vivo to maintain sufficient cardiac output.
\end{abstract}

\section{Introduction}

During radiotherapy of thoracic tumours near the heart, the whole heart or part of the heart may be included in the radiation field. This treatment may lead to functional, histopathological and biochemical changes of the heart $[1,2]$. In the early days of radiotherapy, most of the clinical data on late effects of thoracic irradiation were obtained from post-mortem studies. Nowadays, relevant data on the development of cardiac abnormalities are derived from post-treatment cardiovascular examinations, including radionuclide ventriculography. Thus, patients entering the EORTC H7 and H8 lymphoma trial [3] are subjected to radionuclide angiography in order to evaluate left ventricular ejection fraction (LVEF) before and after completion of radiotherapy and at 1 and 5 years after treatment.

Received 5 July 1996 and in revised form 3 April 1997, accepted 9 June 1997.

Address correspondence to J Wondergem, Department of Clinical Oncology, K1-P, University Hospital Leiden, Rijnsburgerweg 10, 2333 AA Leiden, The Netherlands.
Heart function studies after irradiation, using non-invasive techniques, have been performed in experimental animals, e.g. dogs $[4,5]$, and rodents $[6,7]$. Ex vivo methods to study function loss of rat hearts after local heart irradiation have also been described [8-11]. In vivo measurement of cardiac function in Sprague-Dawley rats hardly showed a reduction in cardiac output or left ventricular ejection fraction within 10 months after a dose of $20 \mathrm{~Gy}[6,7]$. Investigators who studied cardiac function ex vivo by using the isolated working rat heart preparation [8-11], observed a significant decline of cardiac function within 2 months of treatment.

To elucidate the apparent discrepancy between cardiac function parameters obtained in vivo and ex vivo, we firstly examined the time course of haemodynamic changes inflicted by local heart irradiation. Both in vivo (LVEF) and ex vivo parameters (stroke volume and cardiac output) for cardiac function were measured in the same animal. Secondly, the possible relation between in vivo and ex vivo data obtained per individual animal was studied using linear regression analysis. 


\section{Materials and methods}

\section{Animals}

Experiments were performed using mature (12-14 weeks old) female Sprague-Dawley rats (obtained from IFFA/CREDO, Broekman, Someren, The Netherlands) with a body weight of about $250 \mathrm{~g}$. During follow-up the animals were housed two to a cage at a $12 \mathrm{~h}$ light and dark cycle and were fed with standard laboratory chow and water ad libitum. For local irradiation, animals were anaesthetized with Ketamine (intramuscular (im), $40 \mathrm{mg} \mathrm{kg}^{-1}$ body weight; Aesculaap, Boxtel, The Netherlands) and Rompun (subcutaneous (sc), $1.6 \mu \mathrm{g} \mathrm{kg}^{-1}$ body weight; Bayer, Leverkusen, Germany). Control animals were also anaesthetized. Experiments were performed under authorization of the Institutional Animal Welfare Committee according to national regulations.

\section{Irradiation technique}

Details of the irradiation technique have been described earlier [11]. Briefly, irradiation was performed with a Philips RT250 X-ray generator (Eindhoven, The Netherlands) operated at $250 \mathrm{kV}$ and $15 \mathrm{~mA}$. X-rays were filtered with a $0.4 \mathrm{~mm}$ Thoraeus filter resulting in a half-valve layer (HVL) of $2.5 \mathrm{~mm}$ copper. Focus-to-heart distance was $25 \mathrm{~cm}$ and the dose rate was $1.95 \mathrm{~Gy} \mathrm{~min}^{-1}$. The rats were individually irradiated using parallel opposed fields (anteroposterior 1:1). During irradiation the thorax of the rat was shielded with two $4 \mathrm{~mm}$ thick lead plates with holes (diameter $1.9 \mathrm{~cm}$ ) located precisely over the heart.

\section{Radionuclide ventriculography}

At different time points after irradiation, LVEF was determined in vivo using the multiple gated acquisition method. Animals were anaesthetized with Ketamine $\left(40 \mathrm{mg} \mathrm{kg}^{-1}\right.$, im) and Rompun $\left(1.6 \mu \mathrm{g} \mathrm{kg}{ }^{-1}\right.$, sc). Red blood cells were labelled in vivo with technetium- $99 \mathrm{~m}\left({ }^{99} \mathrm{Tc}^{\mathrm{m}}\right)$. To this purpose, stannous pyrophosphate $(50 \mu \mathrm{g}$ in a volume of $0.1 \mathrm{ml}$ ) was injected into the left femoral vein. $10 \mathrm{~min}$ later $100 \mathrm{MBq}{ }^{99} \mathrm{Tc}^{\mathrm{m}}$-pertechnetate in a bolus of $0.1 \mathrm{ml}$ was injected into the right femoral vein. After equilibration of the tracer over the vascular space (approximately $5 \mathrm{~min}$ ), the animals were fixed in a supine position on a Perspex jig and positioned under the gamma camera (Toshiba, GCA-40A; Tokyo, Japan) equipped with a pinhole collimator. The collimator was positioned close to the animal so that the heart was magnified maximally and anterior views were obtained. Images of the electrocardiogram (ECG) gated acquisition were stored in a dedicated computer system (MDS$\mathrm{A}^{2}$, Medical Data Systems, Ann-Arbor, MI, USA) in a $128 \times 128$ matrix. The ECG signal was recorded by three silver electrodes located at the left fore- and hindpaws and the right side of the chest. Each R-R interval was divided in 28 frames and acquisition was continued until 150000 counts were acquired in the first frame. To measure heart rates greater than 250 beats $\min ^{-1}$ (acceptance limit of computer system), the trigger pulse from the ECG monitor was set to have a death time of $300 \mathrm{~ms}$. Therefore, in the period between two trigger pulses, one to two sequential cardiac cycles are recorded in one series of 28 frames. The pre-set number of counts was reached after about $20 \mathrm{~min}$. In this period approximately 3600 trigger pulses were recorded, leading to an acquisition of maximally 7200 heart cycles.

Analysis of recordings with two cycles in one series of 28 frames showed that these two cycles were similar in shape. Thus, irregularities in heart rhythm during the measurements can be ruled out. When a significant portion of the acquired cycles would have come from irregular heart beats, then the second half of the volume curve would have shown a gross deformation due to fall-off. Since this was not seen in our study, it was concluded that single cycles were not superimposed on double cycles.

The left ventricle was outlined using a semiautomatic edge detection program. The resulting regions of interest (ROI) over the left ventricle in each frame of the cardiac cycle (variable ROI) were used to construct a time versus activity curve for the summed cardiac cycles. This curve was corrected for background activity. In such a curve the maximum represents the number of counts in the end-diastolic phase (EDC) and the minimum represents the number of counts at end-systole (ESC). LVEF was calculated by the equation

$$
\mathrm{LVEF}=\frac{\mathrm{EDC}-\mathrm{ESC}}{\mathrm{EDC}} \times 100 \%
$$

\section{Isolated working rat heart preparation}

4 days after radionuclide ventriculography, the heart was excised, arrested in ice-cold $\left(0^{\circ} \mathrm{C}\right)$ perfusion buffer. It was then mounted on the aortic cannula of the perfusion apparatus as described earlier [11]. First, the heart was perfused retrogradely with a modified Krebs-Henseleit bicarbonate buffer $[11,12]$ at $37^{\circ} \mathrm{C}$ with a hydrostatic pressure of $9.0 \mathrm{kPa}$. During this period the left atrium was cannulated for perfusion in the working mode. After $10 \mathrm{~min}$ of retrograde aortic perfusion, the heart was set to the working mode. Peak systolic pressure, perfusate temperature and heart rate were recorded. Aortic flow rate $(\mathrm{AF})$ and coronary flow rate $(\mathrm{CF})$ were measured by timed collection. Cardiac performance was tested by varying left atrial filling pressure $(\mathrm{LAFP}=$ $0-2.5 \mathrm{kPa}$ ) while keeping the aortic pressure at a 
fixed level $(9.0 \mathrm{kPa})$. Impairment of contractile function of the myocardium due to irradiation is associated with a downward shift of the FrankStarling (F-S) curve [11]. To compare the different $\mathrm{F}-\mathrm{S}$ curves, the integral of the whole $\mathrm{F}-\mathrm{S}$ curve was calculated between LAFP values of 0.5 and $2.5 \mathrm{kPa}$. Ex vivo heart function was expressed both as cardiac output $\left(\mathrm{CO}=\mathrm{AF}+\mathrm{CF} ; \mathrm{ml} \mathrm{min}^{-1}\right)$ and as stroke volume per gram of myocardial tissue $\left(\mathrm{SV} ; \mu \mathrm{g} \mathrm{g}^{-1} \mathrm{ww}\right)$. The latter parameter is derived from the $\mathrm{CO}$ and is corrected for radiation- and age-induced differences in heart rate (HR; beats $\min ^{-1}$ ) and heart weight (HW). Stroke volume is calculated by the following equation

$$
\mathrm{SV}=\frac{\mathrm{AF}+\mathrm{CF}}{\mathrm{HR}} \times \frac{1}{\mathrm{HW}}
$$

\section{Data analysis}

The data presented in text and figures are expressed as mean \pm SEM. Statistical differences between groups were calculated with the Student's $t$-test, using SPSS WIN 6.1 (SPSS Ltd, Chicago, IL, USA). A $p$-value $\leqslant 0.05$ was considered to be statistically significant. Correlations were tested by linear regression analysis using the least squares method.

\section{Results}

Up to 14 months post-treatment, local heart irradiation with a dose of $20 \mathrm{~Gy}$ did not lead to cardiac failure or cardiac-related death. These animals did not show any sign of congestive heart failure. Differences in body weights and organ weights between irradiated and control animals were not evident up to 8 months post-treatment. Between 8 and 12 months post-treatment, irradiated animals showed a slightly impaired growth rate resulting in slightly lower measured body weights ( $449 \mathrm{~g}$ versus $500 \mathrm{~g}$ ) and heart weights (1.56 g versus $1.69 \mathrm{~g}$ ). No significant differences between weights of liver (13.5 g versus $15.8 \mathrm{~g}$ ) and lung ( $2.23 \mathrm{~g}$ versus $1.85 \mathrm{~g}$ ) were observed, compared with control rats. From 14 months onwards, irradiated animals began to die. At 16 months, four out of 10 animals had died as a result of $20 \mathrm{~Gy}$. During this period the surviving irradiated animals gained weight (BW increased from $449 \mathrm{~g}$ to $481 \mathrm{~g}$ ), whereas the control animals lost weight (BW decreased from $500 \mathrm{~g}$ to $455 \mathrm{~g}$ ). At 16 months, heart weights (1.84 g versus $1.69 \mathrm{~g})$, liver weights $(15.5 \mathrm{~g}$ versus $12.9 \mathrm{~g})$ and lung weights $(2.30 \mathrm{~g}$ versus $1.90 \mathrm{~g}$ ) of irradiated animals were slightly elevated when compared with the controls. However, none of the observed differences was statistically significant.

The time-related changes of in vivo heart function after irradiation are illustrated in Figure 1. During the observation period of 16 months, the mean

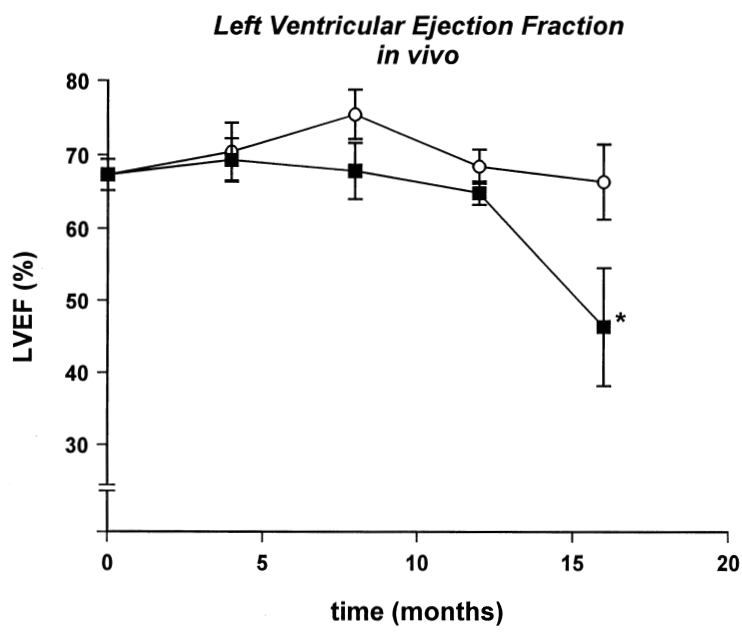

(a)

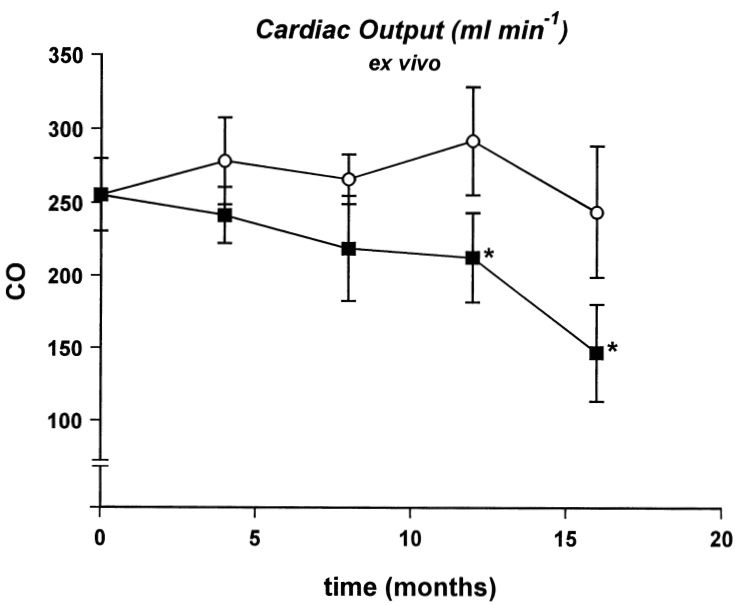

(b)

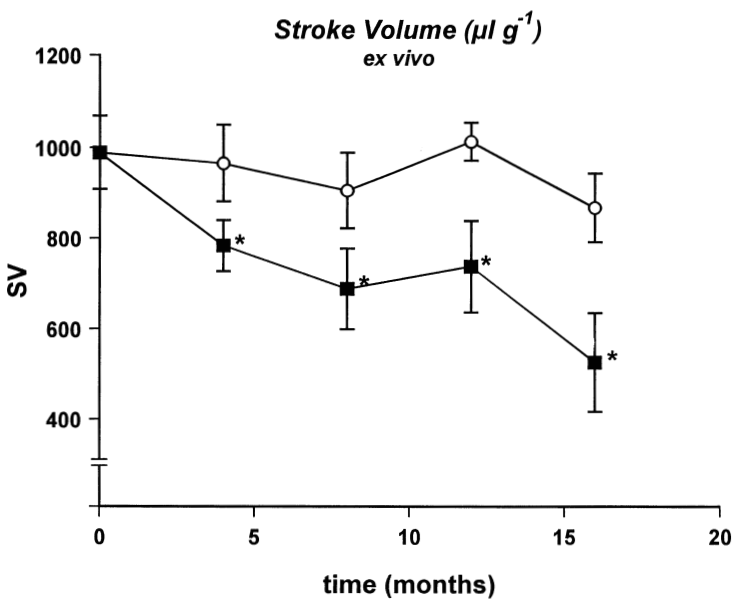

(c)

Figure 1. Time-related changes in cardiac performance after irradiation with a dose of $20 \mathrm{~Gy}$ locally on the heart: $\bigcirc$, age-matched control rats; $\boldsymbol{\square}$, irradiated rats. In vivo cardiac performance is presented as left ventricular ejection fraction (LVEF) using the variable ROI method (see text) (a). Ex vivo cardiac performance measured 4 days after in vivo cardiac performance is presented as cardiac output (CO) (b) and stroke volume (SV) (c). Each point represents the mean of 6-10 animals \pm SEM. *, Significant lower compared with age-matched control rats $(p<0.05)$. 
LVEF calculated for control animals ranged between 66 and 75\% (Figure 1a). After irradiation with a dose of $20 \mathrm{~Gy}$, the LVEF remained stable at a level of about $67 \%$, up to 12 months posttreatment. At 16 months, a sharp decrease of the LVEF was observed to a value of $46 \pm 8 \%$. At this time the LVEF was significantly reduced compared with control values $(p<0.03)$ and with values found 12 months post-irradiation $(p<0.01)$. Heart function parameters measured ex vivo in the same animals, using the isolated working rat heart preparation, demonstrated a different time course of changes after irradiation (Figures $1 \mathrm{~b}$ and $\mathrm{c}$ ). After irradiation, heart function steadily decreased with increasing time interval between treatment and evaluation. At 12 months, the reduction in cardiac output was significant $(p<0.05)$. The decrease of stroke volume per gram of heart tissue was already significant $(p<0.04)$ at 4 months post-treatment. It is evident that after irradiation, cardiac performance measured ex vivo deteriorated more rapidly than cardiac performance measure in vivo (Figures $1 \mathrm{a}, \mathrm{b}$ and $\mathrm{c})$.

During in vivo cardiac performance measurements, the average heart rate of animals treated up to 16 months prior to evaluation was $359 \pm 14$ beats $\min ^{-1}$. Non-treated age-matched control animals presented a heart rate of $377 \pm 12$ beats $\min ^{-1}$. No influence of time interval (between treatment and evaluation) on heart rate was observed.

The relation between in vivo and ex vivo data on heart function obtained from measurements per individual animal was also studied. When tested at 16 months, regression analysis of the pooled data, obtained from irradiated and non-irradiated age-matched control animals was performed. This revealed a significant relation (LVEF versus $\mathrm{CO}$; $p=0.001 ;$ LVEF versus $\mathrm{SV} / \mathrm{g} \mathrm{HW}, p=0.002$ ) between LVEF and cardiac parameters measured ex vivo. However, when tested per treatment group, the earlier observed correlation was lost. Moreover, at all other time points tested $(0,4,8$ and 12 months), no significant relation between in vivo and ex vivo parameters could be found, neither within the different treatment groups nor in the age-matched control groups.

\section{Discussion}

The results of this study demonstrate that local heart irradiation with a dose of $20 \mathrm{~Gy}$ leads to a decrease in cardiac performance. However, the time course of haemodynamic changes after irradiation differs depending on whether cardiac function was assessed from in vivo parameters or from ex vivo parameters. After a dose of $20 \mathrm{~Gy}$, cardiac function measured ex vivo, using the isolated working rat heart preparation, deteriorated more rapidly than the in vivo measured LVEF using radionuclide ventriculography. Up to
12 months post-treatment no decrease in LVEF was observed. Whereas, cardiac output and stroke volume measured ex vivo began to decline within 4 months post-treatment. This observation, together with regression analysis data showing no relation between LVEF and ex vivo parameters of cardiac function, suggests a discrepancy between in vivo and ex vivo parameters measured in this study. Only at 16 months, could a positive relation between both parameters be found. However, at this time point LVEFs of irradiated animals were also depressed. The lack of correlation between the in vivo and ex vivo measurements of cardiac function during the first 12 months post-treatment might be explained by the involvement of compensatory mechanisms being operative in vivo to maintain sufficient cardiac output. Apparently, as long as the heart is adequately compensated, the LVEF does not reflect the true contractile status of the heart, whereas ex vivo parameters do reflect the true contractile status of the heart. When the heart is not adequately compensated, as can be observed at very long time intervals (16 months), LVEF finally drops.

The present findings on rapid impairment of cardiac function measured ex vivo, using the isolated working rat heart preparation, confirm studies previously published by our group [11] and several other institutes [8-10]. In a previous study, using the same rat strain, irradiation caused an early and dose dependent decrease of ex vivo heart function. Benderitter et al [8], using male Wistar rats, already observed a significant reduction (18\%) of the ex vivo cardiac output at 1 month after cardiac irradiation with a dose of $20 \mathrm{~Gy}$ and a further decrease thereafter. An even more prominent decrease of ex vivo heart function was observed by Cilliers et al [9], who used the same rat strain. After a dose of $20 \mathrm{~Gy}$ on the thorax, they observed that the cardiac output fell by $50 \%$ in the first 2 months. Thereafter, cardiac function recovered almost completely. In these latter studies a direct relation between ultrastructural and biochemical changes in the heart and cardiac function were reported [8-10].

The observation that LVEF hardly changed during a 12-month follow-up period is in agreement with data reported by Schultz-Hector et al [6]. These authors studied irradiated SpragueDawley rats (20 Gy to the heart) and did not observe any change in LVEF or cardiac output during the follow-up period of a year. However, Wistar rats developed a decrease in LVEF by $20 \%$ at 80 days post-irradiation and the LVEF remained at this subnormal level until clinical heart failure set in. In another study, using Sprague-Dawley rats, Yeung and Hopewell [7] found that a single dose of $20 \mathrm{~Gy}$ did not cause a change in relative cardiac output in vivo up to 8 months post- 
treatment. As observed in our study, a sharp drop in cardiac output was observed after a long time interval (>10 months).

There is increasing evidence that cardiac damage may develop before clinical symptoms become apparent. Histopathological studies of irradiated rat hearts reveal areas with endothelial enzyme loss, which started at 28 days after a dose of $20 \mathrm{~Gy}$. This was followed by myocardial degeneration at around 70 days [13]. At 6 months after a single dose of $20 \mathrm{~Gy}$ and later, interstitial fibrosis was observed associated with increased expression of TGF- $\beta$ in the subendocardial layers of the left ventricle [14]. Moreover, a depression of myocardial energy metabolism is already evident at 2 months after this radiation dose [15]. Several biochemical studies on the adrenergic system of the heart after irradiation indicate involvement of this system in "cardiac" compensation $[6,16,17]$. The increase in cardiac adrenergic receptor density (both $\alpha$ and $\beta$-receptors) and the decrease of myocardial norepinephrine (NE) concentration following $20 \mathrm{~Gy}$ reflect an increased sympathetic stimulation. Moreover, Persons et al [18] showed an increase of plasma atrial natriuretic peptide (ANP) concentration and ventricular ANP concentration and a decrease of atrial ANP concentration, after irradiation of the heart with $20 \mathrm{~Gy}$. Circulating ANP is known to be involved in the maintenance of sufficient cardiac function as this peptide increases the excretion of sodium and water by the kidneys. Hence, it restores the fluid balance, especially during heart failure [19]. These findings on the involvement of the sympathetic system and ANP after radiotherapy of the heart and the absence of symptoms such as increased liver and lung weights and pleural fluid, suggest that the hearts of the surviving animals are still adequately compensated up to 12 months posttreatment. Thereafter symptoms of clinical heart failure will become evident.

The observation that the differences in LVEF between irradiated animals and age-matched control animals were not significant, up to 12 months post-treatment, might be due to limitations of the various techniques used to detect cardiac disease in a "compensated" heart [6, 7 , present paper $]$. It is common practice in clinical cardiac imaging to acquire the images in a left anterior oblique view so that a good separation between the right and left ventricle is realized. In small animals like rats this is difficult to accomplish since a pinhole collimator is necessary to obtain images that are large enough to distinguish both ventricles during the complete cardiac cycle. Even at maximal magnification, the area of the heart occupied only onetenth of the available matrix. The two reasons mentioned here: (i) a poor separation between the ventricles, and (ii) a small image of the left ventricle, are responsible for a poor delineation of the region of the left ventricle on the end-systolic regions in more than half of the examinations. To improve the accuracy of the data (in particular, outline of left ventricle during the end-systolic phase), a fixed region of interest over the end-diastolic frame was used to generate new time-activity curves based on the original images. This method enabled us to draw an acceptable ROI on the end-diastolic frame in all measurements. When we applied this method, we obtained reliable and reproducible LVEF values in all measurements. It is known, however, that a fixed ROI underestimates the LVEF [20]. This is illustrated in Figure 2 in which the mean $\mathrm{LVEF}_{\text {fixed ROI }}$ calculated for control animals ranged between 45 and $55 \%$. The mean $\mathrm{LVEF}_{\text {variable RoI }}$ varied between 66 and $75 \%$. Calculation of the LVEF, using a fixed ROI instead of a variable ROI, did not lead to a revision of the data. The $\mathrm{LVEF}_{\text {fixed RoI }}$ followed the same pattern of haemodynamic changes after irradiation as described for the $\operatorname{LVEF}_{\text {variable RoI }}$ (Figure 2). Using either method, only at 16 months post-irradiation was a significant reduction of the ejection fraction observed $(p<0.04)$. Using this latter method, no further gain (discrimination between irradiated and non-irradiated hearts) could be reached.

Evaluation of in vivo cardiac function using radionuclide assays has been performed after radiotherapy in several patient studies [1, 2, 21-25]. In the evaluation of left ventricular function different results have been reported. Several authors reported a high incidence of myocardial dysfunction in patients who had no evidence of cardiac disease at the time of evaluation, whereas others were unable to detect any change in left ventricular function after radiotherapy. A review of the literature

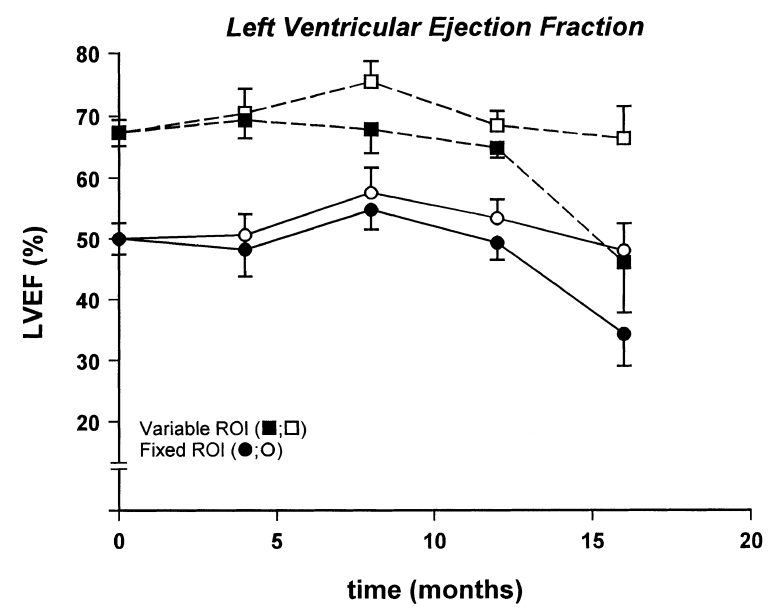

Figure 2. Comparison of the left ventricular ejection fraction calculated using a fixed region of interest $\left(\mathrm{LVEF}_{\text {fixed RoI }} ; \square, \boldsymbol{\square}\right)$ with the left ventricular ejection fraction using a variable region of interest $\left(\mathrm{LVEF}_{\text {variable RoI }} ; \mathrm{O}, \boldsymbol{\bullet}\right)$ in age-matched control rats (open symbols) and in rats irradiated locally on the heart with 20 Gy (solid symbols). 
revealed that several studies reported on patients who were treated for Hodgkin's disease with old radiotherapy techniques and relatively high doses. Moreover, the way authors presented their data on in vivo cardiac performance differed among these studies: in several studies the mean LVEF after radiotherapy did not differ from the mean LVEF in a control group in spite of the fact that several patients had a depressed LVEF $[21,22]$.

Our experimental data show that clinical assessment of radiation effects as measured by LVEF only, might result in an underestimation of the severity of myocardial damage if a damaged heart is being compensated by cardiac and/or circulatory regulation mechanisms. To detect masked cardiac damage in patients after radiotherapy, additional methods are required, such as analysis of myocardial contractility (response to inotropic stimuli), measurements of circulating plasma ANP levels, and determination of the activity of the reninangiotensin-aldosteron system.

\section{Acknowledgments}

We are indebted to $\mathrm{Mr} \mathrm{R}$ Kuit for valuable technical help. This investigation was supported by the Dutch Cancer Foundation, Koningin Wilhelmina Fonds (Grant IKW 90-15) and the Universitair Onderzoeksinstituut voor Radiopathologie en Stralenbescherming (Grants 9.0.2 and 9.0.7).

\section{References}

1. Schultz-Hector S. Radiation-induced heart disease: review of experimental data on dose response and pathogenesis. Int J Radiat Biol 1992;61:149-60.

2. Stewart JR, Fajardo LF, Gillette SM, Constantine LS. Radiation injury to the heart. Int J Radiat Oncol Biol Phys 1995;31:1201-11.

3. Kluin-Nelemans JC, Henry-Amar M, Carde P, et al. Assessment of thyroid pulmonary, cardiac and gonadal toxicity in stages-I-II Hodgkin's disease. In: Proceedings of 3rd International Symposium on Hodgkin's lymphoma, Köln, Germany, 19-23 September 1995.

4. Arom KV, Bishop VS, Grover FL, Trinkle JK. Effect of therapeutic-dose irradiation on left ventricular function in conscious dogs. Ann Thorac Surg 1979;28:166-75.

5. McChesney SL, Gilette E, Orton EC. Canine cardiomyopathy after whole heart and partial lung irradiation. Int $\mathbf{J}$ Radiat Oncol Biol Phys 1988;14:1169-74.

6. Schultz-Hector S, Böhm M, Blöchel A, et al. Radiation-induced heart-disease: Morphology, changes in catecholamine synthesis and content, $\beta$ adrenoceptor density, and hemodynamic function in an experimental model. Radiat Res 1992;129:281-9.

7. Yeung TK, Hopewell JW. Effects of single doses of radiation on cardiac function in the rat. Radiother Oncol 1985;3:339-45.

8. Benderitter M, Maingon P, Abadie C, et al. Effect of in vivo heart irradiation on the development of antioxidant defenses and cardiac functions in the rat. Radiat Res 1995;144:64-72.
9. Cilliers GD, Harper IS, Lochner A. Radiation induced changes in the ultrastructure and mechanical function of the rat heart. Radiother Oncol 1989;16:311-26.

10. Cilliers CD, Lochner A. Radiation-induced damage of the Wistar rat heart: biochemistry and function. Radiother Oncol 1993;27:216-22.

11. Wondergem J, van der Laarse A, van Ravels FJM, et al. In vitro assessment of cardiac performance after irradiation using an isolated working heart preparation. Int J Radiat Biol 1991;59:1053-68.

12. Krebs HA, Henseleit K. Untersuchungen über die Harnstoffbildung im Tierkörper. Zeitsch Zool Physiol Chem 1932;210:33-66.

13. Lauk S. Endothelial alkaline phosphatase activity loss as an early stage in the development of radiation-induced heart disease in rats. Radiat Res 1987;110:118-28.

14. Wondergem J, Weterings ECP, Strootman EG, et al. Fibrosis and transforming growth factor beta (TGF$\beta$ ) in the heart after local heart irradiation. In: Proceedings of 43rd Annual meeting of the Radiation Research Society, San Jose, USA, 1-6 April 1995:p. 180.

15. Franken NAP, Hollaar L, Bosker FJ, et al. Effects of in vivo heart irradiation on myocardial energy metabolism in rats. Radiat Res 1993;134:79-85.

16. Franken NAP, van der Laarse A, Bosker FJ, et al. Time-dependent changes in myocardial norepinephrine concentration and adrenergic receptor density following $\mathrm{X}$-irradiation of the rat heart. Int J Radiat Oncol Biol Phys 1992;24:721-7.

17. Wondergem J, Franken NAP, van der Laarse A, et al. Changes in cardiac performance and sympathetic stimulation during and after fractionated radiotherapy in a rat model. Radiother Oncol 1996;38:33-40.

18. Persons CCM, Franken NAP, Wondergem J. Changes in myocardial and circulating atrial natriuretic peptide following thorax irradiation in the rat. Int J Radiat Biol 1996;70:61-8.

19. Winaver J, Hoffman A, Burnett Jr JC, Haramati A. Hormonal determinants of sodium excretion in rats with experimental high-output heart failure. Am J Physiol 1988;254:R776-84.

20. Walton S. Radionuclide ventriculography. In: Murray IPC, Ell PJ, editors. Nuclear Medicine in Clinical Diagnosis and Treatment, Vol. 2. Edinburgh: Churchill Livingstone, 1994:1069-81.

21. Applefield MM, Wiernik PH. Cardiac disease after radiation therapy for Hodgkin's disease: analysis of 48 patients. Am J Cardiol 1983;51:1679-81.

22. Burns RJ, Bar-Shlomo B-Z, Druck MN, et al. Detection of radiation cardiomyopathy by gated radionuclide angiography. Am J Med 1983; 74:297-302.

23. Gomez GA, Park JJ, Panahon AM, et al. Heart size and function after radiation therapy to the mediastinum in patients with Hodgkin's disease. Cancer Treatment Reports 1983;67:1099-103.

24. Gottdiener JS, Katin MJ, Borer JS, et al. Late cardiac effects of therapeutic mediastinal irradiation. Assessment by echocardiography and radionuclide angiography. N Engl J Med 1983;308:569-88.

25. Morgan GW, Freeman AP, McLean RG, et al. Late cardiac, thyroid, and pulmonary sequelae of mantle radiotherapy for Hodgkin's disease. Int J Radiat Oncol Biol Phys 1985;11:1925-31. 